\title{
MPAB, Un outil pour l'étude et le développement de l'enseignement du contrôle numérique des convertisseurs
}

\author{
Guillaume GATEAU, Ana LLOR, Jérémi REGNIER, Hubert PIQUET \\ Guillaume.Gateau@laplace.univ-tlse.fr \\ Département Génie Electrique / Automatique, 2 rue Camichel, 31071 Toulouse Cedex 7
}

\begin{abstract}
RESUME Cet article présente un système de contrôle numérique (MPAB) dédié à l'enseignement de la commande numérique des convertisseurs statiques. Toujours en forte croissance, l'utilisation de techniques numériques récentes (utilisation de DSP et de FPGA) pour le pilotage des convertisseurs statiques permet un grand nombre de possibilités associé à une grande souplesse d'utilisation. Ces nouvelles solutions numériques apparaissent petit à petit chez les industriels et il est maintenant nécessaire, dans le cycle de formation d'un ingénieur, de connaitre et de maîtriser ces techniques et technologies. Cet enseignement a donc pour objectif de familiariser les étudiants de deuxième et troisième année d'Ecole d'ingénieur (Département Génie Electrique / Automatique à l'ENSEEIHT) avec cette approche par le biais de cas d'études simples, comme la réalisation d'une source de courant contrôlée à l'aide d'une cellule de commutation.
\end{abstract}

Mots clés : Techniques numérique, FPGA, DSP, control des convertisseurs, VHDL

\section{1}

\section{INTRODUCTION}

L'utilisation de techniques numériques pour la commande des convertisseurs statiques est de nos jours une étape essentielle dans le cursus d'un ingénieur en Génie Electrique. L'avènement de ces techniques dans le monde industriel justifie amplement l'introduction de cet enseignement dans le programme des études.

L'objectif est de familiariser les étudiants avec des architectures de type FPGA-DSP à travers le pilotage d'une cellule de commutation à IGBT. Nous souhaitons que l'élève réalise une approche complète sur la mise en œuvre de ce type de solution, en abordant aussi bien les aspects liées à l'informatique industrielle que les aspects plus applicatifs et pratiques liés au système commandé.

Les manipulations sont menées en alternance avec des cours théoriques généraux sur les FPGA, les DSP et les principes de commande d'une cellule de commutation. Nous proposons à l'étudiant de découvrir des notions de programmation en VHDL des FPGA (réalisation d'une MLI intersective et d'une fourchette de courant), de mettre en œuvre la communication avec le DSP, de déterminer et d'implanter un algorithme de régulation. Pour ne pas aborder que les aspects informatique industrielle, et rappeler que notre objectif est de commander un système du Génie Electrique, une part non négligeable du travail est également consacrée aux simulations du comportement électrique de la cellule avec différents modes de pilotage et aux essais avec puissance. Cet enseignement comptabilise $44 \mathrm{~h}$ d'interventions où l'étudiant suivra $\mathbf{6 h}$ de cours théoriques et $\mathbf{3 8 h}$ dispensées sous la forme d'un bureau d'étude.

Après cette introduction permettant de situer le contexte de cet enseignement, la première partie sera consacrée à la présentation des différents principes de contrôle étudiés dans le cadre de la réalisation d'une source de courant, contrôlée par l'utilisation d'une cellule de commutation à deux interrupteurs. La seconde partie présentera le système de contrôle développé pour l'étude des différentes solutions envisagées. La troisième partie sera consacrée à la présentation des deux solutions finalement retenues pour le contrôle, ainsi qu'à la présentation des résultats expérimentaux. L'article se terminera par une conclusion faisant le bilan sur la première année de mise en œuvre de ce nouveau programme d'enseignement.

\section{ETUDE DU PILOTAGE D'UNE CELLULE DE COMMUTATION}

\subsection{Principes généraux}

Nous rappelons dans un premier temps aux étudiants les principales lois de comportement et les principes élémentaires de commande en courant d'une cellule de commutation.

\subsubsection{Rappel sur la cellule de commutation}

La figure 1 montre le schéma de la cellule de commutation utilisée. Les interrupteurs K1 et K2 sont commandés de manière complémentaire, $\alpha$ représentant le rapport cyclique du bras.

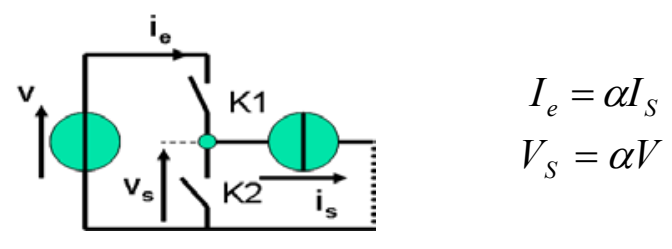

Fig. 1 : Schéma de la cellule de commutation

Comme l'illustre la figure 2, nous proposons, à partir d'un organe de pilotage, de contrôler le courant 
dans l'inductance à la sortie du bras en générant sur les interrupteurs de puissance les ordres appropriés.

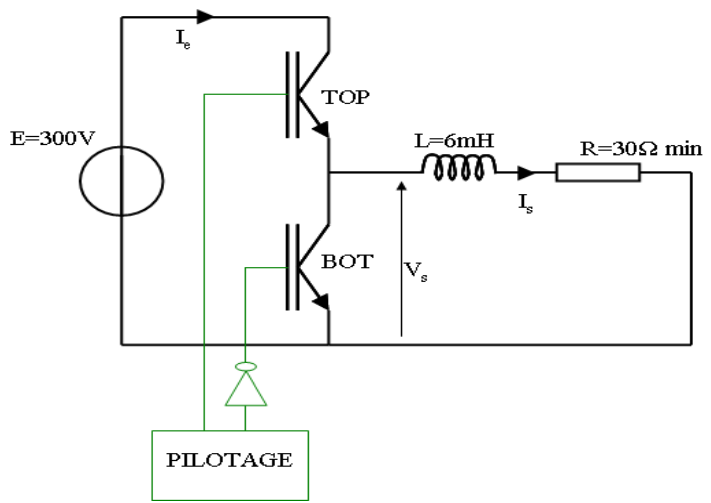

Fig. 2 : Schéma de principe du contrôle du courant dans une cellule de commutation

Plusieurs solutions sont possibles pour ce cahier des charges et nous abordons deux techniques différentes : la commande directe par fourchette de courant et la commande indirecte avec régulateur échantillonné et MLI.

\subsection{Commande Directe : Pilotage par fourchette de courant}

La figure 3 rappelle le principe d'une commande directe par fourchette de courant.

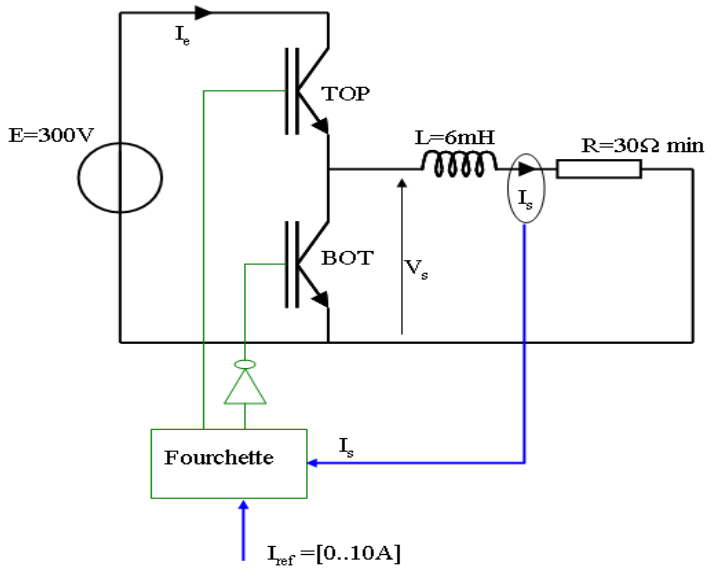

Fig. 3 : Schéma de principe du contrôle direct par fourchette de courant

Il s'agit de maintenir le courant dans une bande délimitée par deux bornes extrémales centrées autour d'un courant de référence $I_{r e f}$. L'amplitude de cette bande, dite bande d'hystérésis, est nommée $\Delta I$. La commande doit s'assurer que le courant reste compris entre $I_{r e f} \pm \Delta I / 2$. L'interrupteur TOP passe à l'état bas quand le courant atteint la borne supérieure, et à l'état haut quand il atteinte la borne inférieure. Il conserve son état à l'intérieur de la bande (voir figure 4).

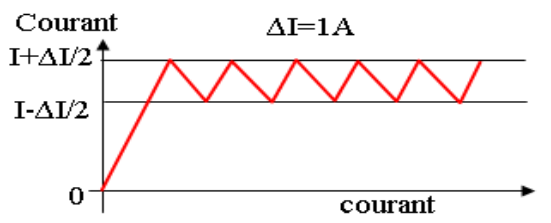

(a) - Allure du courant contrôlé par hystérésis

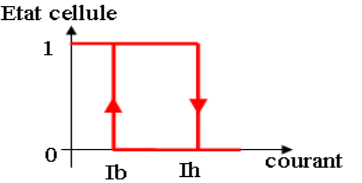

(b) - Gestion de l'état de la cellule

Fig. 4 : Principe de la commande par hystérésis.

\subsubsection{Commande indirecte : Pilotage par MLI et régulateur PI}

La figure 5 rappelle le principe d'une commande indirecte. Le principe de cette commande consiste à utiliser un régulateur PI pour générer le rapport cyclique qui permettra au courant d'atteindre sa valeur de référence. Ce rapport cyclique est ensuite, par l'intermédiaire de la MLI, transformé en ordre de commande pour piloter les interrupteurs de la cellule.

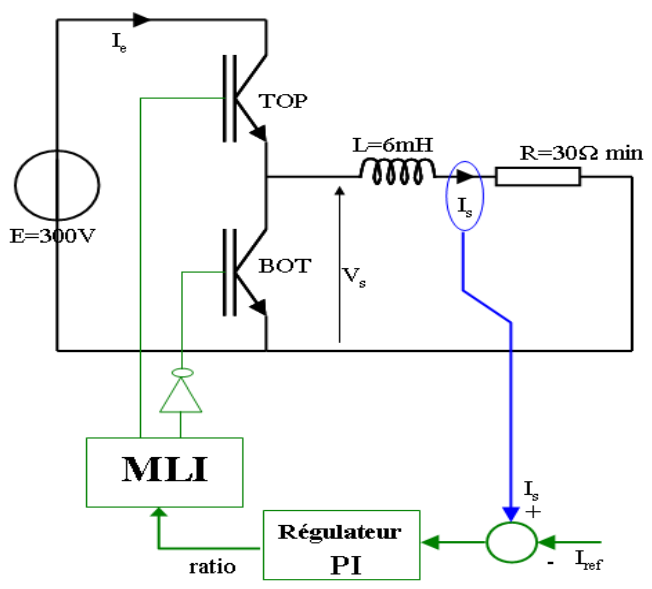

Fig. 5 : Schéma de principe du contrôle indirect par régulateur PI

\subsection{Simulations}

Les différentes stratégies de commande sont simulées avec PSIM pour bien appréhender les aspects fonctionnels de la cellule. Lors de la mise en place sur le support expérimental des stratégies de pilotages, il faudra régler un certain nombre de paramètres (fréquence de découpage, bande passante du régulateur, largeur de la bande d'hystérésis, ...). Cette étape per- 
met aux étudiants de ne pas perdre de vue la finalité de leur travail qui est de commander un système du Génie Electrique, donc de bien en maîtriser les contraintes associées à la transformation de l'énergie par la cellule. Le paramétrage des degrés de liberté de la commande doit être fait en gardant à l'esprit les conséquences de ces choix sur la partie puissance.

\section{PRESENTATION DU SYSTEME DE COMMANDE DEDIE.}

Le système de pilotage est constitué d'un DSP (Texas Instrument TMS320C6713) associé à une carte d'interface permettant, d'une part, de piloter les interrupteurs d'un convertisseur statique (utilisation d'un FPGA ALTERA ACEX1K100), et, d'autre part, de gérer les mesures de tension et de courant provenant du système au moyen de convertisseurs Analogique/Numérique.

La figure 6 nous présente le synoptique de ce système constitué de trois cartes bien distinctes : une carte DSP, une carte fille numérique connectée au DSP et une carte fillette recevant les systèmes de conversion.

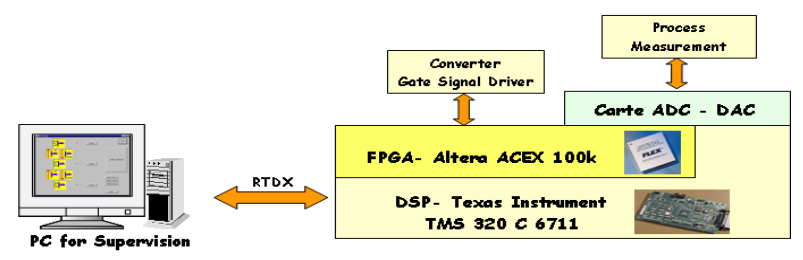

Fig. 6 : Synoptique de la carte dédiée vantes :

Les capacités du système complet sont les sui-

TABLEAU I : CARACTERISTIQUES TECHNIQUES DU SYSTEME

\begin{tabular}{|l|c|}
\hline Horloge DSP & $225 \mathrm{MHz}$ \\
\hline $\begin{array}{l}\text { capacité de calcul en vir- } \\
\text { gule flottante DSP } \\
\text { TMS320C6713 }\end{array}$ & 1350 MFLOPS \\
\hline Mémoire interne DSP & $256 \mathrm{KO}$ \\
\hline $\begin{array}{l}\text { Mémoire externe } \\
\text { SDRAM 100MHz }\end{array}$ & $16 \mathrm{MBytes}$ \\
\hline Connexion PC Hôte & USB 2.0 \\
\hline $\begin{array}{l}\text { Entrée / Sorties Numéri- } \\
\text { ques Bidirectionnelles }\end{array}$ & 24 bits \\
\hline $\begin{array}{l}\text { Entrée / Sorties Numéri- } \\
\text { ques Unidirectionnelles }\end{array}$ & 32 bits \\
\hline $\begin{array}{l}\text { Conv. Ana/ Num (Temps } \\
\text { de conversion 1 us) }\end{array}$ & $2 \times 4$ voies avec sampling \\
\hline $\begin{array}{l}\text { Conv. Ana/ Num (Fmax } \\
=40 \mathrm{MHz} \text { ) }\end{array}$ & $2 \times 1$ voies \\
\hline $\begin{array}{l}\text { Conv. Num/ Ana (setting } \\
\text { time } 100 \mathrm{~ns} \text { ) }\end{array}$ & 2 voies \\
\hline
\end{tabular}

Le synoptique détaillé de cet assemblage est donné en figure 7. On distingue la carte DSP (DSKC6713), la carte FPGA (MPAB_NUM) et la carte de conversion Analogique/Numérique (MPAB_ANA).

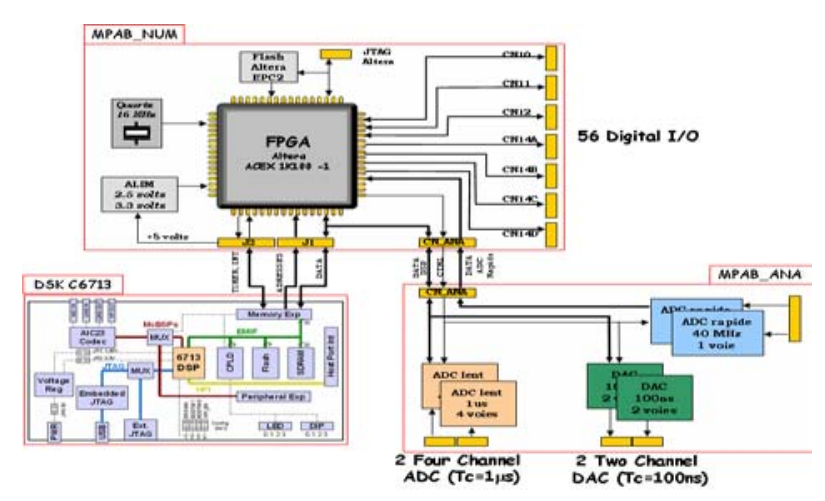

Fig. 7 : Synoptique interne de la carte $M P A B$

Il est important de noter que les convertisseurs analogiques/numériques « rapides » peuvent fonctionner jusqu'à une fréquence de conversion de $40 \mathrm{MHz}$ et sont reliés directement au FPGA. Cela nous permet donc d'envisager la réalisation d'une fourchette de courant numérique, toujours délicate à réaliser. Dans ce cas le DSP peut simplement être utilisé pour effectuer une supervision du montage.

La figure 8 nous montre une photographie du système de commande ainsi obtenu.

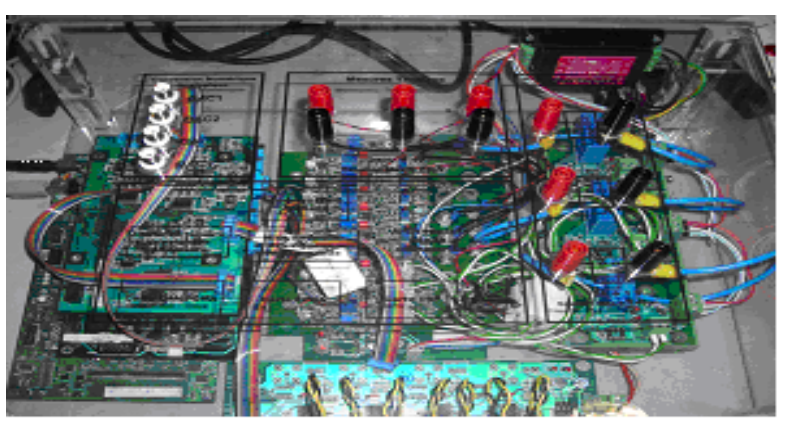

Fig. 8 : Photo de l'environnement de commande numérique proposé

\section{DEVELOPPEMENT DE 2 SOLUTIONS POUR LA REALISATION D'UNE SOURCE DE COURANT}

\subsection{Choix de l'architecture de commande}

Les deux solutions proposées pour la commande de la cellule (directe et indirecte) présentent l'avantage de conduire à deux architectures de commande différentes. La première sera implantée avec seulement le FPGA et seconde mettra en œuvre l'ensemble FGPA-DSP. Une réflexion concernant la 
justification de ce choix s'impose alors : quelle architecture choisir pour quelle application ? Quelles fonctionnalités doivent être gérées par le FPGA, par le DSP ? Nous sensibilisons les étudiants au fait que le FPGA est un circuit câblé, donc plus rapide que le DSP qui fonctionne comme un processeur et doit interpréter des instructions. Ainsi, quand il s'agit d'implanter la commande directe par fourchette ou la MLI, qui sont de simples comparaisons avec des grandeurs de référence, le FPGA est plus approprié. Le DSP est quant à lui mieux adapté à des tâches de calculs numériques et possède des facilités pour gérer les aspects temps réels. Il sera donc associé au FPGA pour mettre en place la régulation de courant indirecte, plus consommatrice d'opérations mathématiques.

\subsection{Fourchette de courant numérique}

L'implantation de la commande directe de courant par utilisation d'une fourchette est réalisée à l'aide d'un composant numérique de type FPGA associé à un convertisseur analogique/numérique (voir figure 9).

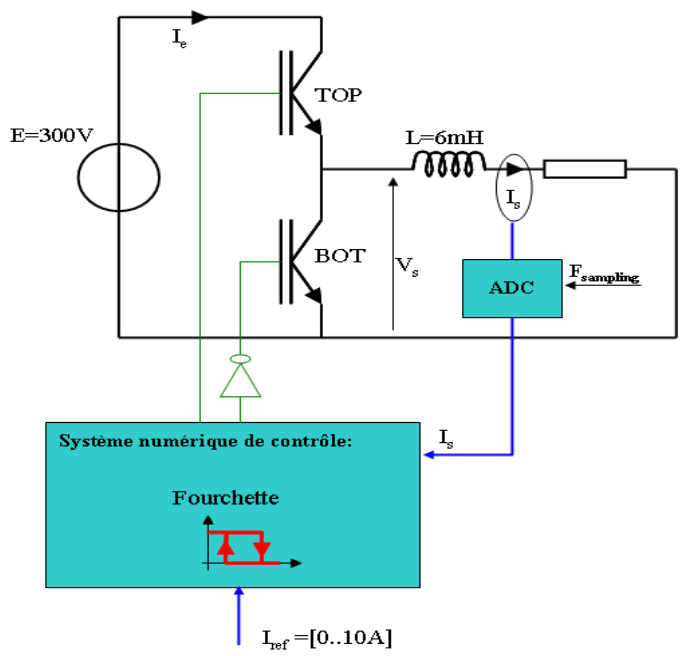

Fig. 9 : Schéma de principe du contrôle indirect par régulateur PI

La programmation s'effectuera en VHDL à l'aide d'un bloc, comme illustré en figure 10. Dans un premier temps, les entrées de courant mesuré et de référence, ainsi comme la largeur de la fourchette (DELTA_I), seront spécifiées directement en VHDL par stockage de valeurs dans des mémoires.

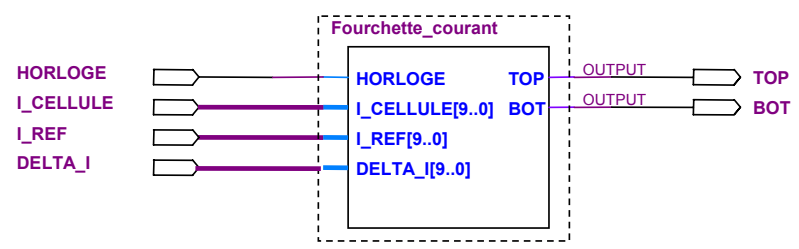

Fig. 10 : Schéma d'implantation de la fourchette de courant en $V H D L$
Les étudiants doivent alors réaliser des simulations fonctionnelles et temporelles sur le schéma proposé afin de comprendre la répercussion des éventuels retards associés à une implantation réelle sur le composant.

Un des problèmes que l'on peut mettre en évidence est l'apparition de rebonds de la commande due aux oscillations du courant mesuré lors des commutations des interrupteurs de puissance. Pour le résoudre on propose aux étudiants d'implanter un compteur à l'intérieur du bloc fourchette qui inhibe la comparaison entre les deux courants pendant un temps légèrement supérieur à un temps mort. De cette façon, on peut s'affranchir des oscillations de courant pendant la phase transitoire.

La deuxième partie de l'étude consiste à faire intervenir le DSP pour la saisie des valeurs de courant de référence et de l'amplitude de la fourchette. Ces valeurs seront passées au FPGA à travers de registres au format adéquat. Le courant mesuré sera obtenu à partir du convertisseur CAN rapide directement connecté au FPGA et fonctionnant à une fréquence de $20 \mathrm{MHz}$

\subsection{Utilisation d'une MLI et d'une régulation de type PI}

\subsubsection{Mise en place de la MLI}

Pour réaliser l'implantation de la MLI, nous proposons aux étudiants de réaliser deux blocs VHDL : un compteur/décompteur pour générer la porteuse et un bloc comparateur qui permet, par comparaison de la porteuse avec la valeur du rapport cyclique à imposer, de générer les ordres de commande de la cellule. Les figures 11 -a et 11-b illustrent les principes de réalisation de la MLI.

Les solutions choisies sont ici encore simulées avec le logiciel QUARTUS pour en vérifier le bon fonctionnement.

\subsubsection{Dimensionnement des régulateurs numéri- ques}

Les étudiants sont dans cette tâche confrontés au problème de dimensionnement et d'implantation d'un régulateur discret. Nous proposons une approche par numérisation d'un correcteur continu et par synthèse en $\mathrm{z}$, dans le but d'obtenir l'équation de récurrence qui fournira la valeur du rapport cyclique. Le comportement de la cellule avec les régulateurs obtenus est simulé ave PSIM. 


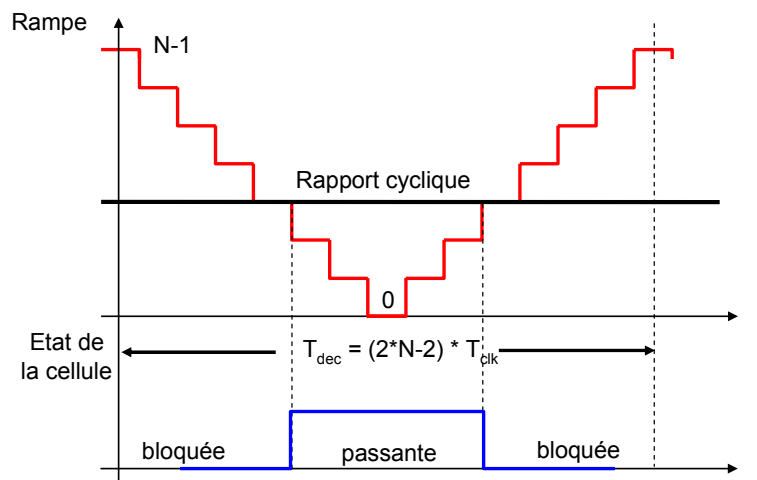

(a) Principe de la MLI

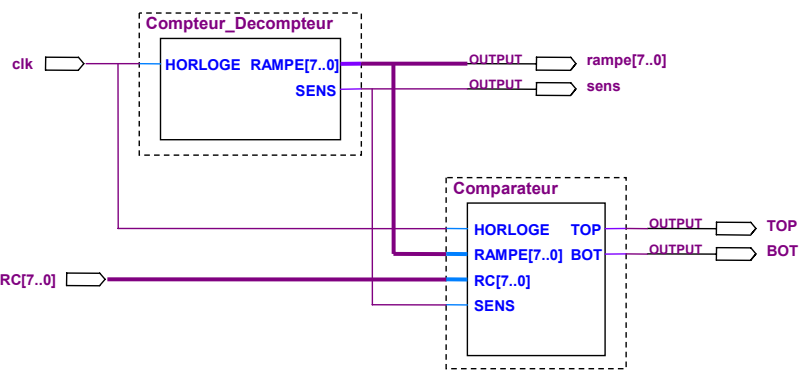

(b) Réalisation VHDL

Fig. 11 : Principe et réalisation en VHDL de la MLI

\subsubsection{Exploitation du lien FPGA-DSP}

Une fois la MLI mise en place et les algorithmes de commande déterminés, nous abordons l'exploitation du lien FPGA-DSP. L'algorithme de pilotage est implanté sur le DSP et fournie à la MLI du FPGA la valeur du rapport cyclique à imposer à la cellule de commutation. Les étudiants doivent alors mettre en place ce lien entre le DSP et le FPGA.

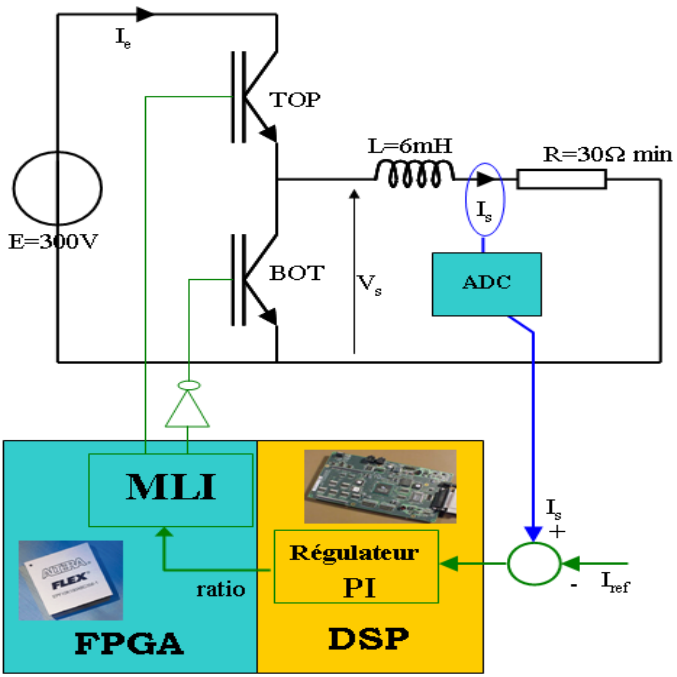

Fig. 92 : Principe du contrôle indirect par régulateur PI
Une prise en main de l'environnement de programmation de la carte DSP (logiciel Code Composer Studio) est tout d'abord proposée. Nous leur faisons découvrir les principes liés à l'utilisation du noyau temps réel de la carte DSP à travers la programmation de timers et la mise en place des fonctions d'interruption logicielles et matérielles.

Une fois ces connaissances acquises, nous leurs proposons de mettre en place la synchronisation DSPFPGA. Ils doivent programmer un des timers du DSP pour cadencer le FPGA qui renvoie un signal d'interruption vers les entrées d'interruption du DSP. Les étudiants doivent alors mettre en place la fonction d'interruption matérielle dans laquelle sera implantée leur algorithme de régulation. L'envoi de la valeur du rapport cyclique à la carte FPGA est réalisé par une écriture dans un registre, ce qui nous permet également d'étudier les mécanismes de dialogue entre les deux organes de commande.

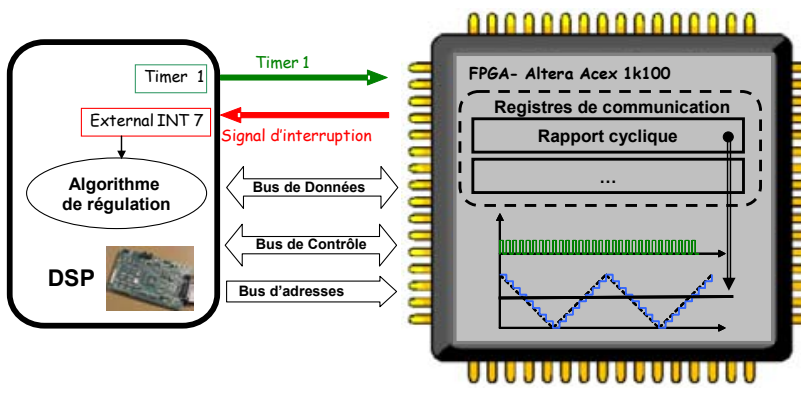

Fig. 103 : Schéma de principe du lien DSP-FPGA

\section{CONCLUSIONS}

Cet enseignement proposé en $2^{\text {ième }}$ année d'école d'ingénieur permet de familiariser les étudiants avec les techniques numériques modernes, mais également de leurs rappeler que le processus sous contrôle reste dans le domaine du génie Electrique et nécessite de la part du concepteur une réelle connaissance de son fonctionnement. Le déroulement proposé sous forme de cours, de BE ainsi que d'essais expérimentaux (figure 14) est un réel succès et réponds parfaitement aux besoins de formation de nos nouveaux ingénieurs.

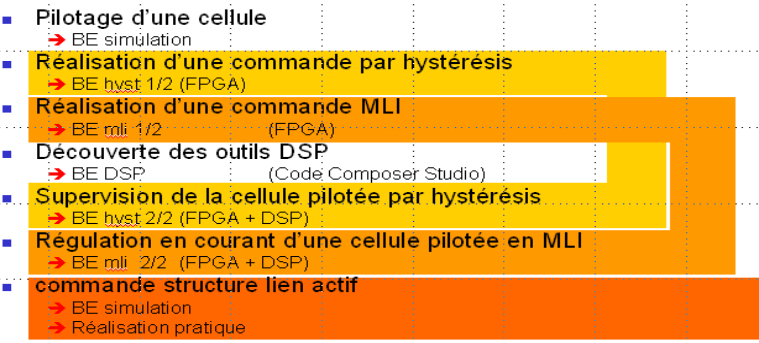

Fig. 14 : Organisation des enseignements 
L'objectif de cette formation est totalement atteint et permet non pas de former des spécialistes du contrôle numérique mais plus des concepteurs, sachant prendre en compte les contraintes d'un cahier des charges complexes que représente la commande des convertisseurs. 